\title{
Income, Poverty and Prices: Comparisons at National and Sub-national Level: Editors' Introduction
}

\author{
Luigi Biggeri $^{1}$ - D. S. Prasada Rao ${ }^{2}$
}

Accepted: 6 February 2017 / Published online: 25 February 2017

(C) Springer Science+Business Media Dordrecht 2017

\begin{abstract}
About half a century ago a small group of researchers at the University of Pennsylvania, headed by Professor Irving Kravis, started an ambitious research program to conduct international comparisons of prices and real incomes across countries. Their project, which started in 1970 with comparisons involving 10 countries, has grown into a major international statistical initiative known as the International Comparison Program (ICP). The ICP is currently a program under the auspices of the United Nations Statistical Commission and conducted jointly by various regional organizations such as the African Development Bank, Asian Development Bank, Eurostat, OECD, Commonwealth of Independent States (CIS) and the Economic Commission for Latin American Countries (ECLAC). The ICP is coordinated by the Global Office located at the World Bank. The coverage of the ICP has expanded significantly over the last five decades and the program is now truly a global program which included 146 countries in its 2005 round and 199 countries in the 2011 round of the ICP.

The ICP is undoubtedly the most reliable source of data on internationally comparable economic aggregates such as the purchasing power parities (PPPs) of currencies gross real domestic product (GDP), per capita consumption expenditure and investment. The most recent set of results for the year 2011 can be found in World Bank (2014). International comparison data generated by the ICP are used for many purposes including comparative assessment of economic performance of countries and regions; measurement of regional and global inequality and poverty; and for the purpose of cross-country comparisons of prices and living standards.
\end{abstract}

\section{Luigi Biggeri}

biggeri@disia.unifi.it

D. S. Prasada Rao

d.rao@uq.edu.au

1 Emeritus Professor of Economic Statistics, Department of Statistics, Informatics and Applications, University of Florence, Viale Morgagni 59, 50134 Florence, Italy

2 School of Economics, The University of Queensland, St. Lucia, QLD 4072, Australia 
A comprehensive account of the framework for the ICP and its implementation, with details on the methods of collection of data and methodologies followed for the computation of the PPP, can be found in World Bank (2013) which is often referred to as the ICP Book.

The growth in the production of international statistics from the ICP and the increasing use of these results in economic and social policy development have led, during the last few decades, serious debate about the need for construction of PPPs at sub-national level. The estimation of sub-national PPPs is increasingly becoming important and is crucial for large countries like China, India, and so on, and in the case of countries (like Italy) where the regions and provinces have different levels of development and cost of living. Moreover, in the recent years there has been a worldwide increase in the demand for household's income, poverty and living conditions estimates at the local (small area) level within regions and provinces that take into account the cost of living differences, since these quantities are crucial in planning local policies aimed at decreasing poverty and social exclusion.

Now a days, it is important to note that more and more academics are carrying out interesting experimental estimations of sub-national PPPs yet up to now few countries have produced official indexes of spatial prices or have carried out experiments to this aim. An off-shoot of this research on sub-national PPPs is the recent research on the possible integration of price data collected for the purpose of the compilation of the consumer price index (CPI) for the purpose of compilation of sub-national and cross-country price comparisons. As yet, the most promising approach is to compute sub-national household consumption PPPs using the data collected by the National Statistical Offices (NSOs) for compiling CPI and the use of Country Product Dummy (CPD) methods.

The main objective of this Special Issue is to bring together several strands of research at the national and sub-national level to the attention of researchers around the world and keep them abreast of the developments in this important area. ${ }^{1}$ The enhanced interest on PPPs and results from the ICP and other estimations of PPPs are not always matched by a proper understanding of the process and methodology used in generating the results. Such an understanding of the methods is necessary to appreciate both the usefulness and the limitations in the use of these results for various purposes.

We have included 11 selected papers, divided into three groups, which provide a background to the process of compilation of PPPs at the global, regional and local level and focusing on the use of these statistics for international and sub-national comparisons and analysis.

The first group consist of four papers, which are devoted to the presentation of the ICP and its evolution, to the improvement of a specific measure for the PPP of the health services and to an interesting application of internationally comparable data on PPP converted real incomes.

We are pleased and excited to head this special issue with a contribution by Alan Heston who is undoubtedly one of the pioneers of international comparisons of prices and real incomes through his work at the University of Pennsylvania along with Irving Kravis and Robert Summers. In addition to the continued involvement in the ICP over the last fifty

\footnotetext{
1 The editors gratefully acknowledge the University Centre and the Bank Etruria of Arezzo, Italy, sponsoring and financing an International Conference on the measurement of PPP at the national and subnational level. Significantly revised versions of a few of the papers presented at that Conference form the core of this Special Issue. We are thankful to the enthusiastic support from the contributing authors in bringing this volume to fruition.
} 
years, Heston was an architect, together with Summers, in the construction of the now famous Penn World Table (PWT). The PWT since its beginnings in the early 1980s has been a major source of panels of PPPs and real aggregates covering the period since 1950s and over 150 countries. The development of PWT has in fact been a major reason behind increased demand of statistics from the ICP. In his contribution to this Issue, Heston provides a historical account to international accounts with a catchy title, the Flaw of One Price, which provides a perspective that contributes to an increased understanding of the evolution of work on international comparisons. Heston discusses also new and old issues in comparisons among and within countries discussing how political issues become intertwined with spatial comparisons at the national and international level.

A natural follow up to Heston's contribution is the paper by Hamadeh (World Bank), Mouyelo-Katoula (World Bank), Paulus Konijn (Eurostat) and Franchette Koechlin (OECD) which describes the current state of play with respect to the methods and uses of ICP. Mouyelo-Katoula as the global manager for ICP (2011) and Nada as the Senior Statistician at the World Bank who is currently leading ICP work at the World Bank are well placed to provide an insider-view of the ICP. The other two contributors, Konijn and Koechlin who have been spearheading ICP activities respectively at the Eurostat and OECD bring their experience and insights into the contribution. The users of PPPs and real economic aggregates from ICP would benefit from further reading of important publications referred to in this contribution. The authors provide an overview of the ICP program as implemented in the 2011 round and walk the reader through the main results from this round and their implications for the world economy and the measurement of inequality and poverty. Moreover, they conclude with thoughts about the future of the ICP underscoring the need for reconciling the results of various ICP rounds.

A major development in the compilation of PPPs within the ICP is the treatment of comparison-resistant services such as education and health. The paper by Koechlin, Konijn, Lorenzoni and Schreyer outlines a new approach to comparison of health service prices across countries. In the past exercises of the ICP, health service comparisons were solely based on the prices of inputs used in delivering health services. These typically would include material inputs such as pharmaceuticals and other consumables; services of medical professionals like doctors, nurses, radiologists; services of non-professionals providing administrative and other support to hospitals and capital equipment. However the use of input-based approach to health services implies zero productivity growth over time and same level of productivity across countries. These assumptions are restrictive and, therefore, the approach outlined in this paper is a major step forward in that it attempts to measure the volume of health services delivered and make price level comparisons for health services. This approach can be extended to comparisons of education and other sectors.

The next paper by Ganegodage, Rambaldi, Rao and Tang presents an interesting application of internationally comparable data on PPP converted real incomes and a raft of socio-economic indicators to construct an index of development. The paper recognizes the multidimensional nature of development and uses factor analysis to identify the factors that can represent various facets of development, by using 42 socioeconomic variables for 129 countries for the 10-year period from 2003 to 2012. Several indices relating to development are computed including an index that captures the role of technology and institutional quality; and another measure that represents basic level of development. These two indices are combined to form the development index, which is then compared to income per capita (PPP adjusted) and the Human Development Index. The authors outline the use of Jackknife approach to test the significance of the extracted loadings. The development index 
proposed here has more power in discriminating between countries at different stages of development and represents a major step forward to such indices discussed in the literature.

The second group of four papers describe methods and present results from efforts around the globe to produce sub-national PPPs for comparing price levels and real expenditures across areas within national boundaries. The paper by Biggeri, Laureti and Polidoro has an excellent review of various studies conducted by national and international statistical organizations and also by individual researchers and in doing so presents an excellent backdrop the remaining papers in this genre. Moreover, in the framework of a new project that the Italian National Statistical Office is implementing, the paper analyses the appropriate use of existing CPI data to compute PPPs at sub-national level. In particular, the CPD method for compiling sub-national PPPs at the basic-heading level (that, in the international comparison literature, is the lowest level of aggregation at which expenditure weights are available) is applied. The paper also explores and implements strategies to account for the presence of spatial correlation in prices of items from different regions of Italy. The paper presents results of various experiments based on price data concerning $7 \mathrm{BHs}$ and 19 Italian regional chief towns, highlighting the performance of different variants of the CPD model and how the type and characteristics of the data affect the estimates obtained. The main conclusion of the paper is that where data are available it is important to use individual price quotations rather than average prices from the regions and that it is important to account for the presence of spatial correlation.

The paper by Aten describes the methodology developed at the Bureau of Economic Analysis (BEA) for the purpose of making price comparisons across different metropolitan areas in the United States of America and computing the price-adjusted estimates of regional personal income. Regional price parities (RPPs) are computed for 38 metropolitan and urban areas of the U.S. using CPI price data as the principal source and data on rents.Then the method is expanded to cover the remaining non metropolitan portion of each state and all counties in the United States. The item level price data are aggregated using a modified version of the weighted Country-Product-Dummy (CPD-W) method enhanced by the inclusion of various hedonic characteristics to account for quality differences that might be reflected in the observed price data. For higher levels of aggregation (several expenditure categories), above the elementary index level, the paper uses the Geary-Khamis method mainly due to the additive consistency property of the index. Results are presented for the years 2012 and 2013. The resulting RPPs reflect the common perception of the different level of price among the different areas showing that large metropolitan areas such California, New York and District of Columbia have higher price levels. However, some of the smaller areas such as New Hampshire, Vermont and Hawaii also show higher price levels mainly reflecting higher rents. Moreover, the relative price level of services tends to increase with increasing incomes and with higher rents, and mirrors the results observed in international comparisons of real incomes and PPPs.

In the paper of Majumder and Ray, the authors used a different approach in the compilation of PPPs. Instead of price data they make use of specific unit values obtained from the information on expenditures and quantities from the National Sample Surveys (NSS) in India. The aggregation methodology is anchored on the CPD method discussed also in Biggeri et al's contribution in this Issue. An important contribution of the paper is to go beyond the usual regression based CPD approach and compute PPPs based on the concept of True Cost of Living Index. The parametric approach, where a particular parametric specification used for the demand system, in the paper is anchored on the Quadratic Almost Ideal Demand System (QAIDS). The methodology used in the paper is then related to some of the known variants of CPD due to Rao (2005), Diewert (2005), and the Hill and Syed 
(2015) system. The paper reports spatial price indices for various provinces or states in India, for three periods and for the rural and urban areas. The empirical contribution includes a systematic comparison between the spatial price indices from alternative models; and the analysis of sensitivity of the spatial price indices to the choice of commodities and estimates of rural-urban differentials in spatial price indices. Readers need to bear in mind that results here are based on unit value prices from household surveys and not on prices collected for the CPI, but the approach followed by the authors is surely of interest for the topic of sub-national PPPs estimations.

Moving the focus from India to another large country, Biggeri, Ferrari and Zhao focus on the estimation of price level differences in China. The authors present a short review of previous studies on the measurement of price level differences across provinces and municipal cities in China. Based on price data for 62 goods and services for 31 Chinese Provinces and Municipal Cities, the authors compute PPPs using the Gini-Elteto-KovesSzulc (GEKS) method which is also the method currently used in the ICP (see World Bank 2013). The necessary weights are obtained from household level data drawn from the Urban and Rural Household Surveys (URHS) of China. The overall consistency of the results obtained is checked by examining the relationship between the provincial and municipal city per capita disposable income and the PPPs and a rough comparison between the PPPs and the spatial price indexes obtained by other authors. Price comparisons based on the full basket indicate that prices in Shanghai are $14.7 \%$ higher than in Beijing. Zeizhang show the highest price level, 49\% higher than Beijing. The paper also presents an in-depth analysis of the results which are also presented for commodity sub-groups.

The last group of three papers focus on comparisons of inequality, household expenditure and poverty at sub-national and local level. The paper by $\mathrm{Wu}$ and Rao focuses on the trends in the inequality of income in China which according the estimation of some authors has risen from 0.30, as measured by the Gini coefficient, to 0.55 in 2012. The paper offers an analytical and empirical framework to study the role of urbanization on inequality. Based on econometrically estimated relationship between inequality and urbanization using data over the period 1987-2010 for 20 provinces collected directly from Chinese Statistical Yearbooks, the authors find a robust inverted-U shaped relationship. Using the relationship, an urbanization rate of 53\% is identified as a threshold after which inequality is likely to decrease, with possible implication that provinces with levels of urbanization higher than the threshold will experience reductions in income inequality. The paper also examines the role of rural-urban wage differential on provincial inequality.

The paper by Marchetti and Secondi consider the important problem of obtaining reliable estimates of household consumption expenditure at the provincial level in Italy which also take account of the different level of prices. The paper makes use of Small Area Estimation (SAE) methods as the Household Budget Surveys do not provide reliable estimates at a disaggregated level. The authors estimate average household consumption expenditure for 109 provinces for the year 2012. The nominal expenditure estimates are adjusted for price level differences across provinces and over time by using and extrapolating Regional PPPs computed by the Italian Statistical Office for the year 2009. In general, prices in Northern cities are higher than those observed in the Centre and in the Southern part of Italy. The main contribution of the paper is that it has provided reliable local-level estimates of household consumption expenditure in real terms which are quite important for policy makers' intervention at national as well as at the local level.

The last paper by Giusti, Masserini and Pratesi is aimed at filling the gap in the availability of reliable estimates of household income and living conditions at the local level, highlighting some key issues and challenges in the analysis of poverty at the local 
level using survey data. The problem is very important because in the last few years there has been a worldwide increase in the demand for poverty and living conditions estimates at the local level, since these quantities can help in planning local policies aimed at decreasing poverty and social exclusion. The paper concentrates on local comparisons of monetary poverty indicators focusing on the adequate SAE techniques to be used due to insufficient sample sizes available. The various problems faced, due to the available data and to the choice of a model for them, are underscored. The authors present estimates of mean household equalized income and the head count ratio poverty measures for small local areas in Tuscany region in Italy for the year 2011, i.e. for 57 Labor Local Systems. The paper also discusses the issues, and present some evidence, arising from the definition and choice of the poverty line to count the poor and the need for adjustments reflecting price level differences across the areas. The PPSs are required but are usually not available at the local level and the paper outlines many open issues in this field indicating possible lines of research.

In conclusion, we enthusiastically recommend the papers in this Special Issue to practitioners involved in making spatial price comparisons across countries and regions within countries. The methods described in these papers represent the current practice and thus provide a blue print for researchers interested in this area. We are pleased to include papers that have dealt with inequality and poverty measurement at the local where it is necessary to use SAE methods. The range and coverage of these papers provide a rich and diverse set of methods and results relevant in this important area of sub-national price, real income, inequality, and poverty comparisons.

\section{References}

Diewert, W. E. (2005). Weighted Country Product Dummy variable regressions and index number formulae. Review of Income and Wealth, 51(4), 561-570.

Hill, R. J., \& Syed, I. (2015). Improving international comparisons of price at basic heading level: An application to the Asia-Pacific region. Review of Income and Wealth, 61(3), 515-539.

Rao, D. S. Prasada. (2005). On the equivalence of the weighted Country-Product-Dummy (CPD) method and the Rao-system for multilateral price comparisons. Review of Income and Wealth, 51(4), 571-580.

World Bank. (2013). Measuring the real size of the world economy: The framework, methodology and results of the International Comparison Program-ICP (p. 650). Washington, DC: World Bank.

World Bank. (2014). Purchasing power parities and the real size of world economies-A comprehensive report of the 2011 International Comparison Program (p. 308). Washington, DC: World Bank. 